\title{
Prevalência autorreferida de hipertensão e fatores associados em comunidades quilombolas do Rio Grande do Sul, Brasil
}

\author{
Self-reported prevalence of hypertension and associated factors in \\ quilombola communities in the State of Rio Grande do Sul, Brazil
}

Sílvia Pauli (http://orcid.org/0000-0002-1470-9171) ${ }^{1}$

Fernanda Souza de Bairros (http://orcid.org/0000-0002-8611-058X) ${ }^{2}$

Luciana Neves Nunes (http://orcid.org/0000-0003-0151-1876) ${ }^{1}$

Marilda Borges Neutzling (http://orcid.org/0000-0003-2276-5197) ${ }^{1}$

${ }^{1}$ Programa de PósGraduação em

Epidemiologia, Departamento de Medicina

Social, Faculdade de Medicina, Universidade Federal do Rio Grande do Sul (UFRGS). R.

Ramiro Barcelos 2400/2, Santa Cecília. 90035-003 Porto Alegre RS Brasil. silviaspauli@gmail.com ${ }^{2}$ Departamento de Assistência e Orientação Profissional, Faculdade de Enfermagem, UFRGS. Porto Alegre RS Brasil.

\begin{abstract}
The study sought to identify the prevalence of arterial hypertension (AH) and possible associated factors in adults residing in quilombola communities in the State of Rio Grande do Sul (RS). A transversal population-based study, carried out in 2011, included 589 adult households in quilombola communities in the State of RS, by sampling proportional to the size. The outcome was obtained by the question: "Has a doctor ever told you that you have hypertension?" Poisson regression with robust variance and hierarchical input variables were used. Finally, the population attributable fractions per component (PAFC) were calculated for modifiable factors associated to $A H$. The self-reported prevalence of $A H$ was $38.3 \%$ (CI95\% 31.4\%-45.1\%). Adjusted analysis revealed an association of the outcome with age group, education, excessive alcohol consumption, waist circumference and the presence of diabetes. $P A F C$ analysis revealed that if the individuals had greater schooling, the prevalence of $A H$ would be reduced. Given the high prevalence of $A H$ and the extreme social vulnerability of this population, public policies that guarantee their access to fundamental rights (health, income and schooling) could have a significant impact in reducing this outcome.
\end{abstract}

Key words Arterial hypertension, Risk factors, Vulnerable communities, African continent ancestry group, Prevalence studies
Resumo Este estudo teve como objetivo identificar a prevalência e fatores associados à hipertensão arterial (HA) em adultos quilombolas do Rio Grande do Sul (RS). Trata-se de um estudo transversal de base populacional, realizado em 2011, com 589 adultos responsáveis por domicílios e amostragem proporcional ao tamanho. $O$ desfecho foi obtido com a pergunta: "Algum médico já lhe disse que você tem hipertensão?". Foi empregada regressão de Poisson, com variância robusta e entrada hierarquizada das variáveis. Por fim, foram calculadas as frações atribuiveis populacionais por componente (FAPC) para fatores modificáveis associados à $H A$. A prevalência autorreferida de HA foi de 38,3\% (IC95\% 31,4\%45,1\%). A análise ajustada revelou associação do desfecho com faixa etária, escolaridade, consumo excessivo de álcool, circunferência da cintura acima do adequado e presença de diabetes. A análise dos FAPC revelou que, se os indivíduos tivessem maior escolaridade, a prevalência de HA seria reduzida. Diante da elevada prevalência de HA e da extrema vulnerabilidade social dessa população, politicas públicas que garantam seu acesso a direitos fundamentais (saúde, renda e escolaridade) poderiam ter impacto importante na diminuição desse desfecho.

Palavras-chave Hipertensão arterial, Fatores de risco, Comunidades vulneráveis, Grupo de ascendência continental africana, Estudos de prevalência 


\section{Introdução}

Pressão arterial (PA) elevada é o maior contribuinte individual para a carga mundial de doenças e à mortalidade global. Segundo a Organização Mundial da Saúde (OMS), em 2013, 9,4 milhões de mortes foram decorrentes da hipertensão e suas complicações ${ }^{1}$. No Brasil, a Pesquisa Nacional de Saúde (PNS) revelou que 21,4\% dos indivíduos com 18 anos ou mais referiram diagnóstico de hipertensão arterial (HA) ${ }^{2}$.

Diferenças na prevalência de HA têm sido encontradas em diversos grupos étnico/raciais em estudos internacionais ${ }^{3}$ e nacionais ${ }^{4}$. Nos Estados Unidos, essas disparidades foram, em grande parte, mediadas por determinantes sociais da saúde ${ }^{5}$. Os determinantes sociais da saúde, bem como os sistemas criados para lidar com doenças, afetam desproporcionalmente HA e doença cardiovascular ${ }^{6}$. No Brasil, além das desigualdades socioeconômicas, fatores relacionados ao racismo e discriminação aumentam a vulnerabilidade em saúde de povos negros e indígenas ${ }^{7}$. A população afro descendente brasileira, discriminada historicamente, possui piores indicadores de renda e escolaridade, está mais exposta às violências, além de o acesso a serviços de saúde ser mais difícil, produzindo, em geral, evolução e prognóstico piores para as doenças que a afetam ${ }^{8}$.

Comunidades quilombolas são núcleos populacionais de afrodescendentes que, diante da condição de escravidão vivida no passado, constituíram formas particulares de organização social e ocuparam espaços geográficos estratégicos no Brasil' ${ }^{9}$, geralmente em áreas rurais com relativo grau de isolamento ${ }^{10}$. Essa população é marcada por processos de discriminação e exclusão que imprimem em sua realidade um quadro socioeconômico bastante excludente em relação à população brasileira de modo geral ${ }^{11}$. A interseccionalidade da raça/etnia, classe social, isolamento geográfico e processo histórico de exclusão social potencializa um quadro desfavorável na situação de saúde dessa população.

Apesar do número expressivo de famílias residentes em comunidades quilombolas no Brasil (mais de 3000 comunidades no território nacional certificadas até agosto de $2017^{12}$ ), verifica-se que há poucos estudos com amostras representativas ou de base populacional nas unidades federativas brasileiras, de modo a permitir o conhecimento e comparação da situação de saúde quilombola no país, permanecendo essa uma lacuna importante na literatura sobre esta parcela da população brasileira.
No que se refere à hipertensão, recentes estudos realizados com adultos em comunidades quilombolas da Bahia, Minas Gerais, Maranhão, Mato Grosso, Espírito Santo e Alagoas revelaram que a prevalência da doença era elevada, variando de $23 \%$ a $52,5 \%$, e em todos os estudos a população apresentou baixa escolaridade e pertencia às classes econômicas inferiores ${ }^{13-20}$. Tendo em vista o contexto de vulnerabilidade social e a magnitude que representa a HA, todos os esforços devem ser feitos a fim de viabilizar estudos direcionados ao conhecimento deste agravo também em comunidades quilombolas do Sul do Brasil. Dessa forma esse estudo tem o objetivo de identificar a prevalência e analisar os fatores associados à HA em adultos responsáveis por domicílios de comunidades quilombolas do RS.

De acordo com as diretrizes e objetivos da Política Nacional de Saúde Integral da População $\mathrm{Negra}^{21}$, os dados do presente estudo podem contribuir para conferir visibilidade às iniquidades que atingem a população quilombola, identificar necessidades de saúde e, assim, contribuir com o planejamento, avaliação e monitoramento de ações e programas de saúde dirigidos a essas comunidades no Brasil.

\section{Métodos}

\section{Delineamento e amostra}

Em 2011 foi conduzido um estudo transversal de base populacional nas comunidades quilombolas do RS, com o objetivo avaliar a insegurança alimentar (IA). Segundo a Fundação Cultural Palmares, até outubro de 2010, havia no estado 122 comunidades certificadas, nas quais residiam cerca de 3100 famílias. Neste projeto, para o cálculo do tamanho amostral levou-se em consideração a prevalência de IA moderada e grave na população negra do RS, que, segundo a PNAD 2009, era de 9,5\%. Foi estabelecido um erro aceitável de 3 pontos percentuais, efeito de delineamento de 1,5, nível de confiança de 95\%, resultando em uma de amostra de 576 indivíduos. Foram acrescidos $10 \%$ para compensar possíveis perdas e recusas, totalizando 634 famílias de 22 comunidades quilombolas, rurais e urbanas, de 17 municípios do RS. Neste estudo, tendo como foco a prevalência de HA, que é de 22,7\% de acordo com o Vigitel, o cálculo do tamanho amostral indica que uma amostra de 571 indivíduos garante uma margem de erro de 3,8 pontos percentuais para um nível de confiança de 95\%, efeito de delineamento de 1,5 e considerando 
uma população de tamanho conhecido de 3100 famílias.

O processo de amostragem foi realizado em três etapas, pois o número de famílias em cada comunidade diferia de 4 a 275. Etapa 1 - seleção das comunidades a serem incluídas no estudo: atribuía-se um peso a cada quilombo, proporcional ao número de famílias cadastradas. Assim, uma comunidade com 100 famílias tinha 10 vezes mais chance de ser incluída do que outra com 10 famílias. Etapa 2 - definição do número de famílias a serem entrevistadas em cada comunidade: amostragem proporcional ao tamanho de cada comunidade. Etapa 3 - definição dos domicílios a serem contatados: de posse das listas com nomes das famílias pertencentes à comunidade realizou-se amostragem aleatória simples. Entrevistou-se um indivíduo adulto ( $\geq 18$ anos de idade) por domicílio, auto declarado responsável pela família.

\section{Descrição da população do estudo}

No RS, é possível identificar algumas regiões com grande concentração de quilombos rurais, tais como: litoral (municípios de Osório, Mostardas e Palmares do Sul); região central (municípios de Restinga Seca, Formigueiro e entorno); e principalmente região sul (municípios de São Lourenço, Canguçu e Pelotas). A região metropolitana de Porto Alegre abriga sete quilombos urbanos. As principais dificuldades enfrentadas pelas comunidades quilombolas rurais são de ordem socioeconômica, como dimensão reduzida das terras ocupadas (muitas vezes íngremes e de solo pedregoso) e falta de alternativas para a geração de renda. Além disso, grande parte situa-se em locais de difícil acesso: pequenas estradas de chão batido, muitas vezes distantes do transporte público. Em algumas comunidades inexistem estratégias de saúde da família, fazendo com que a população tenha de se deslocar quilômetros em busca de atendimento.

Até outubro de 2010, o estado do Rio Grande do Sul possuía 122 comunidades quilombolas certificadas, estimando-se 3101 famílias $^{12}$. Grande parte dessas comunidades estão localizadas na metade sul do estado, região de menor desenvolvimento socioeconômico.

\section{Coleta de dados e variáveis estudadas}

Características demográficas (sexo, idade, raça/cor, local de moradia), socioeconômicas (renda e escolaridade), de estilo de vida (con- sumo de álcool e tabagismo), diabetes mellitus (DM), transtornos mentais comuns (TMC) e circunferência da cintura (CC) foram avaliadas por meio de questionário padronizado com 96 questões e de medidas antropométricas. A variável idade foi organizada em três categorias: 1839; 40-59 e $\geq 60$ anos e a cor - auto referida - foi dicotomizada em branca e não branca. Para a categorização da variável renda utilizou-se salários mínimos nacionais $(>1 ; 1 / 2-1 ; 1 / 4-1 / 2$; e até $1 / 4)$ vigentes em 2011. A escolaridade, segundo anos completos de estudo foi agrupada em 4 categorias (<1 ano; $1-3$; 4-7 e 8-11 anos). O consumo de álcool foi mensurado a partir do questionário que avaliava tipos de bebidas (cerveja, cachaça, vinho, vodka, whisky, outras) doses consumidas e frequência de consumo de bebidas alcoólicas de cada sujeito.

A partir da informação sobre doses consumidas de cada bebida, foi calculada a quantidade total de álcool consumida em gramas, sendo considerada excessiva a partir de $30 \mathrm{~g} /$ dia para homens e $15 \mathrm{~g} /$ dia para mulheres ${ }^{22}$. O tabagismo foi categorizado em fumante atual (independente do número de cigarros), ex fumante e não fumante. Presença de DM foi verificada através da pergunta "Algum médico já lhe disse que você tem diabetes (açúcar alto no sangue)?” e os TMC identificados pelo 20-item Self-Reporting Questionnaire (SRQ20), utilizando-se o ponto de corte de 8 ou mais repostas positivas para classificação de $\mathrm{TMC}^{23}$. A CC foi aferida por entrevistadores treinados, com fita métrica inextensível (precisão de $1 \mathrm{~mm}$ ), no ponto médio entre a borda inferior da última costela e a crista ilíaca e classificada em adequado e acima do adequado, segundo critérios da $\mathrm{OMS}^{24}$.

O desfecho do presente estudo foi investigado por meio da pergunta: "algum médico já lhe disse que você tem hipertensão (pressão alta)?”. O questionário padronizado, contendo questões socioeconômicas, demográficas, biológicas, de insegurança (Escala Brasileira de Insegurança Alimentar- EBIA) e consumo alimentar (recordatório de 24hs) foi construído especificamente para essa população. Utilizou-se questões amplamente usadas em outros estudos epidemiológicos. Sua compreensão foi testada em duas comunidades quilombolas (uma rural e uma urbana) não selecionadas para o estudo. Após essa etapa foi realizado estudo piloto, a fim de evitar/minimizar possíveis erros na coleta de dados. Todos os entrevistadores participaram de treinamento teórico-prático de 20 horas. Um supervisor esteve em contato permanente com os entrevistadores 
no trabalho de campo e realizou controle de qualidade, por meio de revisitas a $10 \%$ da amostra.

\section{Análise estatística}

Os dados foram duplamente digitados no programa Epidata, com checagem automática de consistência e amplitude. Para as análises estatísticas utilizou-se o software SPSS 16.0 e Stata 8.0. Sendo este um estudo transversal, a fim de evitar a superestimação das medidas de efeito (razões de prevalência) que seria ocasionada por uma regressão logística (cuja medida de efeito é Odds Ratio), uma vez que o desfecho apresenta prevalência alta $(\geq 10 \%)$, foi utilizada a Regressão de Poisson com variância robusta. Levou-se em conta o plano amostral complexo, ou seja, o ajuste dos modelos considerou o efeito de delineamento, corrigindo as medidas de efeito, com os respectivos intervalos de confiança de $95 \%$. O recurso computacional utilizado para o ajuste dos modelos com o efeito do plano amostral complexo foi a função svy do Stata. A entrada das variáveis no modelo multivariável foi de acordo com o modelo conceitual apresentado na Figura 1 , gerando um modelo que pode se chamar de modelo hierárquico. Todas as variáveis de cada

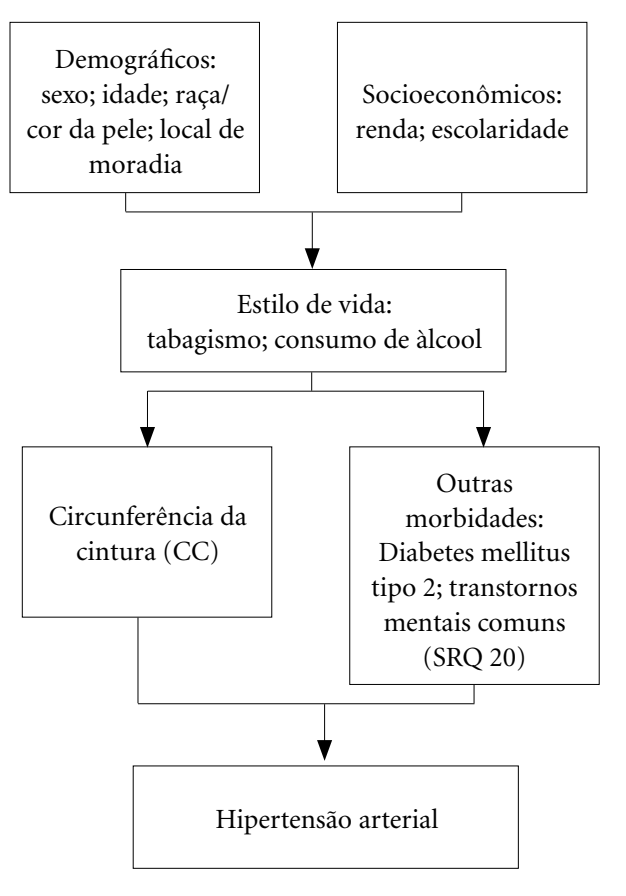

Figura 1. Modelo Hierarquizado de Análise. bloco foram ajustadas em relação umas às outras e as que apresentaram associação ao nível de $20 \%$ $(\mathrm{p} \leq 0,2)$ foram selecionadas para permanecer no modelo seguinte. Dessa forma, as variáveis do bloco 2 foram ajustadas de acordo com as variáveis elegíveis do bloco 1 e, por fim, foi incluído o último grupo de variáveis (bloco 3), atingindo, assim, a definição dos fatores associados com HA. No modelo final, a significância estatística foi estabelecida em 5\%. Cabe mencionar que a estratégia de seleção das variáveis do modelo hierárquico de análise usado nesse estudo centrou-se em dados de ampla revisão da literatura e variáveis disponíveis no banco de dados do estudo realizado em 2011. Por fim, para os fatores modificáveis associados, foram calculadas as frações atribuíveis populacionais por Componentes (FAPC), que considera as relações entre os fatores de exposição e seus confundidores, determinando a proporção total da prevalência do desfecho na população, atribuída a cada componente de exposição. Cada FAPC foi definida conforme Camey et al. ${ }^{25}$ (Figura 2). Conforme a fórmula utilizada, é necessário o cálculo do Risco Relativo (RR), definido pelo quociente da incidência nos indivíduos expostos ao fator pela incidência nos não-expostos. Em estudos transversais, o risco relativo passa a ser chamado como razão de prevalências (RP). Assim, no presente estudo estimamos o quociente da prevalência nos expostos ao fator pela prevalência nos não expostos.

$$
\begin{aligned}
& F A P C_{A B}=\frac{n_{111}}{n_{111}+n_{101}+n_{011}+n_{001}} \times \frac{R R_{A B}-1}{R R_{A B}} \\
& F A P C_{A}=\frac{n_{101}}{n_{111}+n_{101}+n_{011}+n_{001}} \times \frac{R R_{A}-1}{R R_{A}} \\
& F A P C_{B}=\frac{n_{011}}{n_{111}+n_{101}+n_{011}+n_{001}} \times \frac{R R_{B}-1}{R R_{B}}
\end{aligned}
$$

Figura 2. Fórmula para cálculo das Frações Atribuíveis Populacionais por Componente (FAPC).

$\mathrm{FAPC}_{\mathrm{AB}}$ : Fração Atribuível Populacional da componente exposição simultânea aos fatores A e B; FAPC : Fração Atribuível Populacional da componente exposição apenas ao

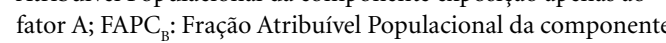
exposição apenas ao fator $\mathrm{B} ; \mathrm{n}_{111}$ : expostos a ambos os fatores; $\mathrm{n}_{101}$ : expostos apenas ao fator $\mathrm{A} ; \mathrm{n}_{011}$ : expostos apenas ao fator $\mathrm{B} ; \mathrm{n}_{001}$ : não expostos; RR: Risco Relativo. 


\section{Aspectos éticos}

O projeto original e o presente estudo foram aprovados pelo Comitê de Ética em Pesquisa da Universidade Federal do Rio Grande do Sul. Foram respeitadas as diretrizes preconizadas pela resolução 466 de 2012.

\section{Resultados}

Foram entrevistados 589 adultos responsáveis por domicílios de 22 comunidades quilombolas do RS. Ocorreu perda de 7\% nas entrevistas em relação à amostra originalmente prevista devido a não localização da família. Não houve recusas. Não há disponibilidade de informações para caracterizar as perdas.

A prevalência de HA auto referida foi de $38,3 \%$ (IC95\% 34,4\%-42,3\%). A maioria dos responsáveis pelo domicílio era do sexo feminino $(64,9 \%)$, residia na área rural $(81,7 \%)$, era de raça/cor não branca $(90,7 \%)$, recebia até $1 / 2$ salário mínimo $(63,3 \%)$ e, em torno de $80 \%$, tinha menos de 8 anos de estudo. A média de idade foi de 45 anos (DP \pm 17$)$, variando entre 18 e 104 anos. O consumo excessivo de álcool foi de $8,2 \% \mathrm{e}$ a prevalência de tabagismo foi de $29,7 \%$. Com relação à presença de outras morbidades, 29,3\% da amostra apresenta TMC e 9,2\% DM (Tabela 1).

A Tabela 2 apresenta os efeitos brutos e ajustados de cada variável independente sobre a HA. De acordo com o modelo hierárquico de análise proposto, todas as variáveis socioeconômicas e demográficas foram selecionadas para continuarem nas análises, exceto raça/cor e renda. Para o segundo modelo, às variáveis selecionadas a partir do modelo 1 foram acrescidas tabagismo e consumo excessivo de álcool. Todas as variáveis permaneceram para a análise no modelo 3 e foram acrescidos os fatores CC, presença de TMC e de DM.

No modelo final, após ajuste para as demais variáveis, escolaridade e consumo excessivo de álcool permaneceram inversamente associadas à $\mathrm{HA}$, e maior idade, CC acima do adequado e presença de DM mostraram-se fatores de risco para o desfecho.

Foram estimadas as FAPC dos fatores modificáveis escolaridade (dicotomizada em $<8$ anos e $\geq 8$ anos de estudo) e CC, que permaneceram associados à HA no modelo final (Tabela 3). A análise das FAPC demonstrou que a exclusão dos fatores baixa escolaridade $(<8$ anos de estudo) e CC acima do adequado, concomitantemente,
Tabela 1. Características demográficas, socioeconômicas, de estilo de vida, circunferência da cintura, presença de transtornos mentais comuns e diabetes em responsáveis de famílias de comunidades quilombolas do Rio Grande do Sul, Brasil, 2011.

\begin{tabular}{|c|c|c|c|}
\hline Variáveis & $\mathbf{N}$ & $\%$ & IC 95\% \\
\hline \multicolumn{4}{|l|}{ Sexo $[\mathrm{n}=589]$} \\
\hline Masculino & 207 & 35,1 & $31,4-39,1$ \\
\hline Feminino & 382 & 64,9 & $60,9-68,6$ \\
\hline
\end{tabular}

Idade $[\mathrm{n}=589]$

18 a 39 anos

40 a 59 anos

$\geq 60$ anos

Raça / Cor [ $\mathrm{n}=589]$

Branca

Não branca

Local de moradia $[\mathrm{n}=589]$

Urbano

Rural

Escolaridade [ $\mathrm{n}=589]$

Menos que um ano

1 a 3 anos

4 a 7 anos

8 a 11 anos

Renda [ $\mathrm{n}=589]$

Mais de 1 salário mínimo

De 1/2 a 1 salário mínimo

De $1 / 4$ a $1 / 2$ salário mínimo

Até $1 / 4$ de salário mínimo

Consumo excessivo de álcool

no último ano $^{*}[\mathrm{n}=585]$

Sim

Não

Tabagismo $[\mathrm{n}=585]$

Nunca fumou

Ex-fumante

Fumante

Transtornos mentais comuns

(SRQ 20) [n = 581]

$\operatorname{sim}$

não

Circunferência da Cintura

$[\mathrm{n}=544]$

Adequada

Acima do adequado

Presença de Diabetes

Mellitus $[\mathrm{n}=585]$

Sim

Não

Presença de Hipertensão [n

$=585$ ]

Sim

Não

*Consumo excessivo de álcool = média diária acima de $30 \mathrm{~g} /$ dia de álcool para homens e $15 \mathrm{~g} /$ dia de álcool para mulheres. 
Tabela 2. Regressão de Poisson com variância robusta para razão de prevalência de hipertensão em responsáveis por famílias residentes em comunidades quilombolas do Rio Grande do Sul.

\begin{tabular}{|c|c|c|c|c|c|c|}
\hline Variáveis & $\begin{array}{c}\text { Bloco 1 } \\
\text { RP (IC95\%) }\end{array}$ & $\mathbf{P}$ & $\begin{array}{c}\text { Bloco 2 } \\
\text { RP (IC95\%) }\end{array}$ & $\mathbf{P}$ & $\begin{array}{c}\text { Bloco 3 } \\
\text { RP (IC95\%) }\end{array}$ & $\mathbf{P}$ \\
\hline \multicolumn{7}{|l|}{ Sexo } \\
\hline Masculino & 1 & & 1 & & 1 & \\
\hline Feminino & $1,41(1,16-1,72)$ & 0,02 & $1,42(1,14-1,78)$ & 0,003 & $0,99(0,79-1,24)$ & 0,896 \\
\hline \multicolumn{7}{|l|}{ Idade } \\
\hline 18 a 39 anos & 1 & & 1 & & 1 & \\
\hline 40 a 59 anos & $3,31(2,29-4,78)$ & $<0,001$ & $3,30(2,25-4,85)$ & $<0,001$ & $2,95(2,01-4,31)$ & $<0,001$ \\
\hline$\geq 60$ anos & $3,49(2,16-5,65)$ & $<0,001$ & $3,15(1,92-5,19)$ & $<0,001$ & $2,73(1,68-4,45)$ & $<0,001$ \\
\hline \multicolumn{7}{|l|}{ Raça / Cor } \\
\hline Branca & 1 & & - & - & - & - \\
\hline Não branca & $1,11(0,61-2,00)$ & 0,722 & - & - & - & - \\
\hline \multicolumn{7}{|l|}{ Local de moradia } \\
\hline Urbano & 1 & & 1 & & 1 & \\
\hline Rural & $1,29(1,08-1,54)$ & 0,007 & $1,30(0,98-1,74)$ & 0,071 & $1,09(0,76-1,57)$ & 0,622 \\
\hline \multicolumn{7}{|l|}{ Renda } \\
\hline Mais de $1 \mathrm{SM}^{\star}$ & 1 & & - & - & - & - \\
\hline De $1 / 2$ a $1 \mathrm{SM}^{*}$ & $1,15(0,90-1,46)$ & 0,250 & - & - & - & - \\
\hline De $1 / 4$ a $1 / 2 \mathrm{SM}^{*}$ & $1,00(0,72-1,38)$ & 0,996 & - & - & - & - \\
\hline Até $1 / 4$ de $\mathrm{SM}^{*}$ & $1,04(0,79-1,38)$ & 0,746 & - & - & - & - \\
\hline \multicolumn{7}{|l|}{ Escolaridade } \\
\hline$<1$ ano & 1 & & 1 & & 1 & \\
\hline 1 a 3 anos & $0,72(0,51-1,01)$ & 0,057 & $0,72(0,51-1,02)$ & 0,060 & $0,67(0,47-0,95)$ & 0,025 \\
\hline 4 a 7 anos & $0,70(0,50-0,98)$ & 0,037 & $0,67(0,47-0,96)$ & 0,030 & $0,61(0,44-0,84)$ & 0,005 \\
\hline 8 a 11 anos & $0,74(0,57-0,96)$ & 0,024 & $0,72(0,51-1,00)$ & 0,053 & $0,62(0,45-0,87)$ & 0,008 \\
\hline \multicolumn{7}{|l|}{ Tabagismo } \\
\hline Nunca fumou & - & - & 1 & & 1 & \\
\hline Ex-fumante & - & - & $1,25(0,96-1,62)$ & 0,090 & $1,18(0,86-1,60)$ & 0,286 \\
\hline Fumante & - & - & $0,86(0,66-1,11)$ & 0,240 & $0,93(0,72-1,20)$ & 0,557 \\
\hline \multicolumn{7}{|l|}{$\begin{array}{l}\text { Consumo excessivo de } \\
\text { álcool }^{* *}\end{array}$} \\
\hline Não & - & - & 1 & & 1 & \\
\hline Sim & - & - & $0,60(0,40-0,91)$ & 0,020 & $0,52(0,31-0,85)$ & 0,013 \\
\hline \multicolumn{7}{|l|}{$\mathrm{CC}^{* * *}$} \\
\hline Adequado & - & - & - & - & 1 & \\
\hline Acima do adequado & - & - & - & - & $1,74(1,41-2,15)$ & $<0,001$ \\
\hline \multicolumn{7}{|l|}{$\mathrm{TMC}^{\star * * *}$} \\
\hline Não & - & - & - & - & 1 & \\
\hline Sim & - & - & - & - & $1,03(0,79-1,33)$ & 0,833 \\
\hline \multicolumn{7}{|l|}{$\mathrm{DM}^{* * * * *}$} \\
\hline Não & - & - & - & - & 1 & \\
\hline Sim & - & - & - & - & $1,38(1,17-1,64)$ & 0,001 \\
\hline
\end{tabular}

Tabela 3. Estimativas das Frações Atribuíveis Populacionais por Componente (FAPC).

\begin{tabular}{lc}
\hline \multicolumn{1}{c}{ FAPC } & Estimativas da FAPC \\
\hline FAPC $_{\mathrm{AB}}$ (escolaridade e CC) & $28,6 \%$ \\
FAPC $_{\mathrm{A}}$ (escolaridade) & $22,5 \%$ \\
FAPC $_{\mathrm{B}}$ (CC) & $7,6 \%$ \\
\hline
\end{tabular}

reduziria $28,6 \%$ da $\mathrm{HA}\left(\mathrm{FAPC}_{\mathrm{AB}} 28,6 \%\right)$. Excluindo apenas o fator baixa escolaridade, diminuiria 22,5\% $\left(\right.$ FAPC $\left._{\mathrm{A}} 22,5 \%\right)$ da HA contra 7,6\% $\left(\right.$ FAPC $\left._{B} 7,6 \%\right)$ se apenas presença de CC acima do adequado fosse excluída. Ou seja, se todos os quilombolas tivessem escolaridade $\geq 8$ anos, a prevalência de HA passaria de $38,3 \%$ para $29,6 \%$. 


\section{Discussão}

Os principais resultados revelaram que a prevalência autorreferida de HA é elevada em adultos de comunidades quilombolas no RS $(38,3 \%)$. Essa prevalência é semelhante àquela verificada pela OMS, em 2011, para adultos maiores de 25 anos vivendo na região das Américas $(35 \%)^{1}$. No entanto, bem superior aos achados de amplos estudos nacionais que, similarmente, utilizaram como desfecho a HA autorreferida. Segundo o Vigitel (inquérito telefônico de vigilância para fatores de risco para doenças crônicas), em 2011 a frequência de HA autorreferida era de 22,7\% para o Brasil como um todo e $29,2 \%$ para Porto Alegre (capital do RS) ${ }^{26}$. Em 2013, a PNS mostrou que $21,4 \%$ dos adultos brasileiros referia presença de $\mathrm{HA}^{2}$. Por outro lado, Bezerra e colaboradores, estudando populações quilombolas de Vitória da Conquista, Bahia (onde a pressão arterial foi diretamente aferida), observaram que quase metade $(45,4 \%)$ dos indivíduos apresentava o desfecho ${ }^{14}$.

É possível supor que, embora validada para a identificação de $\mathrm{HA}$ em estudos populacionais ${ }^{27}$, a medida autorreferida é conservadora, isto é, subestima a prevalência do desfecho, principalmente em populações vulneráveis, com dificuldade de acesso a serviços de saúde. Cabe ressaltar que não há unidades de saúde na maioria das comunidades quilombolas estudadas, ou há falta de profissionais de saúde nas poucas existentes, fazendo com que grande parte dos indivíduos precisem percorrer longas distâncias até a unidade mais próxima. Muitos não possuem meio de transporte próprio e outras formas de transporte também são escassas (em muitas comunidades, há transporte coletivo, mas a frequência não é diária e é necessário pagar passagem). Em um estudo transversal realizado com 358 indivíduos com 18 anos ou mais, no sudoeste da Bahia, Brasil, a prevalência de desconhecimento da HA foi de $44,1 \%$. Entre os indivíduos que realizaram consulta médica nos últimos 12 meses, observouse menor desconhecimento da doença ${ }^{28}$. Dessa forma chama a atenção o fato da hipertensão nas populações quilombolas do RS ser quase o dobro daquela observada em outros estudos nacionais. Uma possível explicação seria a extrema vulnerabilidade social (baixa renda e escolaridade, isolamento geográfico, etc.), a que são submetidas essas populações. Quanto ao isolamento geográfico, $81,7 \%$ residem em zona rural, porém, apesar de no primeiro modelo da análise multivariável a variável "local de moradia" ter se mostrado associada ao desfecho $(p=0,007)$, seu efeito foi diminuído após a inclusão das variáveis intermediárias e proximais a tal ponto que deixou de ser estatisticamente significativa.

A situação de vulnerabilidade dos quilombolas do RS é caracterizada, entre outros, pela baixa renda e baixo nível de escolaridade da maioria dos adultos, além do isolamento geográfico dessa população, já que a grande maioria reside na área rural, longe das cidades e com meios de transportes precários. A população quilombola ainda luta por igualdade de direitos, pela posse e regularização fundiária de suas terras e pela equidade na saúde pública, está ligada a trabalhos rurais, ou culturas de subsistência, e muitos dependem de programas de transferência de renda ${ }^{29}$. A situação de vulnerabilidade das populações quilombolas é confirmada em outros estudos de diferentes regiões do país, representada por baixa classe econômica, baixo nível de escolaridade e acesso restrito à assistência à saúde ${ }^{13-20}$. O fato de a maioria dos responsáveis pelo domicílio serem mulheres pode ser devido à autodeclaração, já que não foi definido de antemão um critério para responsável por domicílio (por ex. maior renda, maior escolaridade, etc.). O critério para essas mulheres serem consideradas as responsáveis pelos domicílios pode ter sido por estas passarem mais tempo em casa (inclusive estavam na hora das entrevistas), pois muitas vezes os homens passam dias fora a trabalho e quem responde pela casa e pelos filhos são elas.

Quanto aos fatores associados à HA, após ajuste para as demais variáveis, escolaridade e consumo excessivo de álcool permaneceram inversamente associadas, e maior idade, CC acima do adequado e presença de DM mostraram-se fatores de risco para o desfecho.

Assim como em outros estudos ${ }^{14,26,27,30}$, observou-se que a frequência de HA aumentava com a idade. A elevação da PA após a idade de 60 anos é consistente com o aumento da rigidez das grandes artérias ${ }^{31}$. É importante chamar a atenção para o fato de que nesse estudo foram encontradas elevadas prevalências de diagnóstico médico prévio de HA referido por indivíduos economicamente ativos $(50,5 \%$ dos indivíduos na faixa etária de 40 a 59 anos referiu ser hipertenso). Isso demonstra a magnitude da doença entre a população quilombola do RS, trazendo a preocupação com possíveis repercussões na saúde e qualidade de vida desses indivíduos e suas famílias.

A escolaridade, um dos determinantes do status socioeconômico, revelou-se importante fator protetor da HA. Resultados semelhantes foram 
verificados em 2013 pela $\mathrm{PNS}^{2}$, isto é, a proporção de indivíduos que referia diagnóstico de HA reduzia à medida que aumentava a escolaridade. O baixo nível de escolaridade (em torno de $36 \%$ tinha menos de 4 anos de estudo) é condizente com o encontrado em um estudo realizado em 2006, em 60 comunidades quilombolas do Brasil, onde $47 \%$ dos chefes de família estudou até o quarto ano do ensino fundamental ${ }^{11}$. Em 2010, estudo de revisão revelou evidências de que o status socioeconômico está inversamente relacionado à exposição a fatores de risco físicos e sociais ${ }^{32}$. Conforme Cois \& Ehrlich $^{33}$, além dos fatores modificáveis que afetam os níveis de PA estarem frequentemente distribuídos de forma desigual entre os estratos socioeconômicos, maior consciência de hipertensão, acessibilidade e adesão ao tratamento e menos estresse crônico podem ser possíveis mediadores do efeito protetor de status socioeconômico mais elevado sobre a doença. Indivíduos com maior escolaridade provavelmente tenham mais acesso e entendimento de informações, melhores condições para praticar o auto-cuidado, o que pode refletir em um melhor estado de saúde.

Diversos estudos têm evidenciado associação entre consumo de álcool e HA, sendo que o efeito varia conforme o gênero e a quantidade e frequência de ingestão ${ }^{34-36}$. Diferentemente, no presente estudo, indivíduos expostos ao consumo excessivo referiram menor prevalência do desfecho. Resultados semelhantes foram revelados em um estudo com população quilombola de Vitória da Conquista (Bahia $)^{14}$. A hipótese de causalidade reversa, isto é, indivíduos com diagnóstico prévio de hipertensão estarem referindo menor consumo de álcool - já indicada no estudo de Bezerra et al. ${ }^{14}$ pode ser utilizada no presente estudo. No entanto, entende-se que estudos futuros devam aprofundar análises quantitativas e qualitativas em relação a essa variável, já que em revisão de literatura, $\mathrm{Pu}-$ ddey \& Beilin ${ }^{35}$ alertam que a magnitude de qualquer efeito protetor nos estudos analisados parece ter sido exagerada em razão de fatores de confusão não medidos, especialmente dieta, estilo de vida e padrões de consumo de álcool.

No que se refere à CC, observou-se a prevalência de HA 74\% maior em indivíduos com CC inadequada, sendo esse resultado muito semelhante ao encontrado na população quilombola de Vitória da Conquista, BA ${ }^{14}$. O tecido adiposo sintetiza e secreta vários mediadores e citocinas, que participam de mecanismos que levam à dislipidemia, resistência à insulina, hipertensão e aterosclerose ${ }^{37}$.
A presença de DM foi associada a uma prevalência $38 \%$ maior de HA na população quilombola do RS. Esse achado é compatível com outros estudos $^{30,38,39}$. O aumento do influxo celular de cálcio pela insulina pode estar relacionado com o desenvolvimento da HA na presença da resistência à insulina, que pode levar à hipertensão por reduzir a produção e liberação do óxido nítrico e aumentar a responsividade a hormônios vasopressores. A hiperinsulinemia e hiperleptinemia aumentam a atividade simpática e consequentemente a reabsorção de sódio e água ${ }^{40}$.

Apesar de não ter sido associada ao desfecho no presente estudo, considera-se que a variável raça/cor mereça atenção. HA é mais prevalente e grave em populações negras em comparação com brancos, porém, os mecanismos exatos não são totalmente compreendidos, dada a etiologia multifatorial da hipertensão essencial e as condições socioeconômicas díspares entre negros e brancos ${ }^{41}$. As estimativas de hereditariedade da HA estão na faixa de $30-60 \%$ e a grande maioria dos casos é "hipertensão essencial", resultante de múltiplos fatores genéticos e ambientais ${ }^{42}$. Há insuficiência de provas para teste com único marcador genético ou epigenético afim de fornecer uma avaliação do risco genético simples para hipertensão essencial ${ }^{43}$. Estudo que investigou potenciais fatores de risco genéticos associados à hipertensão essencial em populações de remanescentes de quilombos do Vale do Ribeira, São Paulo, indica a existência de uma etiologia poligênica na regulação da PA e o risco de HA nessa população, e também que a PA é um fenótipo com elevada herdabilidade ${ }^{44}$.

Outra possível explicação, ainda pouco estudada em estudos epidemiológicos no Brasil, para a maior prevalência de $\mathrm{HA}$ em populações negras seria o fator racismo. Chor e Lima ${ }^{45}$ sugerem que a discriminação racial encontra-se na origem de grande parte das diferenças socioeconômicas que se destacam entre as possíveis causas das desigualdades étnico raciais em saúde. Em estudos futuros, considera-se importante incluir essa variável.

Há uma possível dificuldade desse estudo em observar diferenças entre as categorias, já que a prevalência de não brancos $(90,7 \%)$ é muito superior em relação à de brancos $(9,3 \%)$. A amostra do presente estudo é homogênea também em relação ao status socioeconômico, independente de brancos ou não brancos.

A análise das FAPC mostrou que se os fatores baixa escolaridade e CC acima do adequado fossem excluídos, concomitantemente, $28,6 \%$ da HA seria reduzida. Excluindo apenas o fator bai- 
xa escolaridade, isoladamente, diminuiria 22,5\% do desfecho, ao passo que eliminando apenas o fator CC acima do adequado, haveria 7,6\% de redução na prevalência da doença. Sendo assim, sugere-se que sejam reforçadas as políticas públicas e demais ações que melhorem o acesso dessa população às escolas, já que um percentual considerável da hipertensão poderia ser evitado se todos os indivíduos dessa amostra tivessem 8 anos ou mais de estudo (apenas 20\% apresentou esse nível de escolaridade). Apesar de a FAPC da CC ter sido mais baixa, considera-se que a mesma mereça atenção, por se tratar de uma variável modificável com grande potencial para intervenção no nível de atenção primária à saúde.

Por fim, algumas limitações devem ser consideradas. A primeira refere-se ao delineamento transversal, que não permite que se façam inferências causais, devido a problemas relacionados à temporalidade. A segunda refere-se ao fato de o desfecho ser autorreferido, pois essa medida pode ser influenciada pelo acesso e uso de serviços de saúde. Dessa forma, em uma população com difícil acesso a serviços de saúde, como é o caso no presente estudo, a prevalência da doença pode ser subestimada. Outra limitação refere-se ao tamanho da amostra, que no estudo original foi planejada para identificar a prevalência de IA. Dessa forma, considerando que a prevalência de HA é maior do que a prevalência de IA, o número de indivíduos avaliados deveria ser maior para manter a margem de erro originalmente prevista (3 pontos percentuais). Então, ausência de associações entre HA e algumas variáveis do presente estudo podem ter ocorrido por falta de poder estatístico. Uma última limitação diz respeito à utilização de banco de dados, oriundo de pesquisa planejada para outro desfecho, impossibilitan- do a inclusão de fatores associados à hipertensão importantes e já conhecidos, como por exemplo, dieta, atividade física e uso de medicação, devido a não coleta ou limitações na obtenção das informações. Todavia, como esse foi o primeiro estudo de base populacional com amostra representativa da população quilombola do estado, entende-se que as informações proporcionadas sejam úteis, pois chamam a atenção para a alta prevalência de HA entre adultos quilombolas do RS, uma população por muito tempo ignorada e para a qual não existe informação alguma sobre a situação de saúde e seus determinantes.

De acordo com os resultados deste estudo, a prevalência de HA entre adultos responsáveis por domicílios de comunidades quilombolas do RS é mais elevada do que aquela verificada na população brasileira de modo geral, ou seja, pelo menos no que tange à hipertensão, a situação é pior nessa população. O presente estudo contribui para o preenchimento de uma lacuna do conhecimento a respeito da situação da HA na população quilombola do estado e, além disso, traz conhecimentos sobre alguns determinantes dessa doença. Dessa forma, com dados de uma amostra representativa da população quilombola gaúcha, contribui para compor o quadro nacional. Há ganhos significativos, tanto de saúde quanto econômicos, ligados a detecção precoce, tratamento adequado e bom controle da hipertensão ${ }^{1}$. Os resultados fornecem uma base para o desenvolvimento de políticas públicas que promovam a garantia de acesso a direitos básicos dos seres humanos, como saúde (ampliação e garantia do acesso aos serviços de saúde para ações de prevenção e tratamento adequados, por exemplo) e educação, que como visto é um FAPC importante para o desfecho.

\section{Colaboradores}

S Pauli: Concepção, análise estatística dos dados, interpretação dos dados, escrita, aprovação final. FS Bairros: Concepção, aquisição dos dados, revisão crítica com conteúdo intelectual importante, aprovação final. LN Nunes: Análise estatística dos dados, interpretação dos dados, revisão crítica com conteúdo intelectual importante, aprovação final. MB Neutzling: Concepção, aquisição dos dados, revisão crítica com conteúdo intelectual importante, aprovação final. 


\section{Referências}

1. World Health Organization (WHO). A global brief on hypertension: Silent killer, global public health crisis. World Health Day 2013. [acessado 2017 Fev 8]. Disponível em: http://apps.who.int/iris/bitstream/10665/79059/1/WHO_DCO_WHD_2013.2_ eng.pdf?ua $=1$

2. Instituto Brasileiro de Geografia e Estatística (IBGE). Pesquisa Nacional de Saúde 2013: percepção do estado de saúde, estilos de vida e doenças crônicas. Rio de Janeiro: IBGE; 2014.

3. Kurian AK, Cardarelli KM. Racial and ethnic differences in cardiovascular disease risk factors: a systematic review. Ethn Dis 2007; 17(1):143-152.

4. Barros MBA, César CLG, Carandina L, Torre GD. Desigualdades sociais na prevalência de doenças crônicas no Brasil, PNAD-2003. Cien Saude Colet 2006; 11(4):911-926.

5. Rodriguez F, Ferdinand KC. Hypertension in minority populations: new guidelines and emerging concepts. Adv Chronic Kidney Dis 2015; 22(2)145-153.

6. Ferdinand KC, Nasser AS. Understanding the importance of race/ethnicity in the care of the hypertensive patient. Curr Hypertens Rep 2015; 17(3):15.

7. Schmidt MI, Duncan BB, Azevedo e Silva G, Menezes AM, Monteiro CA, Barreto SM, Chor D, Menezes PR. Chronic non-communicable diseases in Brazil: burden and current challenges. Lancet 2011; 377(9781):1949-1961.

8. Brasil. Ministério da Saúde (MS). Manual de doenças mais importantes, por razões étnicas, na população brasileira afro-descendente. Brasília: MS; 2001. (Série A. Normas e Manuais Técnicos; n.123).

9. Parodi TC. Equidad en salud: una mirada desde la perspectiva de la etnicidad [versión preliminar]. Washington: OPS/OMS; 2001.

10. Silva DO, Gerrero AFH, Gerrero CH, Toledo LM. A rede de causalidade da insegurança alimentar e nutricional de comunidades quilombolas com a construção da rodovia BR-163, Pará, Brasil. Rev. Nutr. 2008; 21(Supl.):83s-87s.

11. Brasil. Ministério do Desenvolvimento Social e Combate à Fome (MDS). Políticas Sociais e Chamada Nutricional Quilombola: estudos sobre condições de vida nas comunidades e situação nutricional das crianças. Cadernos de Estudos Desenvolvimento Social em Debate 2008; 9:142.

12. Brasil. Certificações quilombolas atualizadas [database on the Internet]. 2012 [acessado 2010 Out 20]. Disponível em: http://www.palmares.gov.br.

13. Amorim MM, Tomazi L, Silva RAA, Gestinari RS, Figueiredo TB. Avaliação das condições habitacionais e de saúde da comunidade quilombola Boqueirão, Bahia, Brasil. Biosci J 2013; 29(4):1049-1057.

14. Bezerra VM, Andrade ACS, César CC, Caiaffa WT. Comunidades quilombolas de Vitória da Conquista, Bahia, Brasil: hipertensão arterial e fatores associados. Cad Saude Publica 2013; 29(9):1889-1902.

15. Ferreira HS, Silva WO, Santos EA, Bezerra MKA, Silva BCV, Horta BL. Composição corporal e hipertensão arterial: estudo comparativo envolvendo mulheres das comunidades quilombolas e da população geral de Alagoas, Brasil. Rev. Nutr. 2013; 26(5):539-549.
16. Velten APC, Moraes AN, Oliveira ERA, Melchiors AC, Secchin CMC, Lima EFA. Qualidade de vida e hipertensão em comunidades quilombolas no norte do Espírito Santo, Brasil. Rev. Bras. Pesq. Saúde 2013; 15(1):9-16.

17. Santos EC, Scala LCN, Silva AC. Prevalência de hipertensão arterial e fatores de risco em remanescentes de quilombos, Mato Grosso, Brasil. Rev Bras Hipertens 2015; 22(3):100-105.

18. Barbosa MCL, Barbosa JB, Guerra LFA, Barbosa MFL, Barbosa FL, Barbosa RL, Guida DL, Martins MLB, Bouskela E, Nascimento MDSB, Melo GSO, Castro MMS. Dislipidemia e risco cardiovascular em afrodescendentes: um estudo em comunidades quilombolas do Maranhão, Brasil. Rev Bras Med Fam Comunidade 2015; 10(36):1-10.

19. Oliveira SKM, Pereira MM, Guimarães ALS, Caldeira AP. Autopercepção de saúde em quilombolas do norte de Minas Gerais, Brasil. Cien Saude Colet 2015; 20(9):2879-2890.

20. Silva TSS, Bomfim CA, Leite TCR, Moura CS, Belo NO, Tomazi L. Hipertensão arterial e fatores associados em uma comunidade quilombola da Bahia, Brasil. Cad. Saúde Colet. 2016; 24(3):376-383.

21. Brasil. Ministério da Saúde (MS). Política Nacional de Saúde Integral da População Negra: uma política para o SUS. 2a ed. Brasília: Editora do Ministério da Saúde; 2013.

22. Moreira LB, Fuchs FD, Moraes RS, Bredemeier M, Duncan BB. Alcohol intake and blood pressure: the importance of time elapsed since last drink. J Hypertens 1998; 16(2):175-180.

23. Mari JJ, Williams P. A validity study of a psychiatric screening questionnaire (SRQ-20) in primary care in the city of São Paulo. Br J Psychiatry 1986; 148:23-26.

24. World Health Organization (WHO). Waist Circumference and Waist-Hip Ratio. Geneva: WHO; 2011. (Report of a WHO Consultation).

25. Camey SA, Agranonik M, Radaelli J, Hirakata VN. Fração Atribuível Populacional. Rev. HCPA 2010; 30(1):77-85.

26. Brasil. Ministério da Saúde (MS). Vigitel Brasil 2011: vigilância de fatores de risco e proteção para doenças crônicas por inquérito telefônico. Brasília: MS; 2012. [Série G. Estatística e informação em saúde]

27. Chrestani MAD, Santos IS, Matijasevich AM. Hipertensão arterial sistêmica auto-referida: validação diagnóstica em estudo de base populacional. Cad Saude Publica 2009; 25(11):2395-2406.

28. Bezerra VM, Andrade ACS, César CC, Caiaffa WT. Desconhecimento da hipertensão arterial e seus determinantes em quilombolas do sudoeste da Bahia, Brasil. Cien Saude Colet 2015; 20(3):797-807.

29. Freitas DA, Caballero AD, Marques AS, Hernández CIV, Antunes SLNO. Saúde e comunidades quilombolas: uma revisão da literatura. Rev CEFAC 2011; 13(5):937-943.

30. Oliveira BFA, Mourão DS, Gomes N, Costa JMC, Souza AV, Bastos WR, Fonseca MF, Mariani CF, Abbad G, Hacon SS. Prevalência de hipertensão arterial em comunidades ribeirinhas do Rio Madeira, Amazônia Ocidental Brasileira. Cad Saude Publica 2013; 29(8):1617-1630. 
31. Franklin SS, Gustin W4th, Wong ND, Lasson MG, Weber MA, Kannel WB, Levy D. Hemodynamic patterns of age-related changes in blood pressure - the Framingham Heart Study. Circulation 1997; 96(1):308-315.

32. Evans GW, Kim P. Multiple risk exposure as a potential explanatory mechanism for the socioeconomic status-health gradiente. Ann NY Acad Sci 2010; 1186:174-189.

33. Cois A, Ehrlich R. Analysing the socioeconomic determinants of hypertension in South Africa: a structural equation modelling approach. BMC Public Health 2014; 14:414.

34. O'Keefe JH, Bybec KA, Levie CJ. Alcohol and cardiovascular health: the razor-sharp double-edged sword. J Am Coll Cardiol. 2007; 50(11):1009-1014.

35. Puddey IB, Beilin LJ. Alcohol is bad for blood pressure. Clin Exp Pharmacol Physiol 2006; 33(9):847-852.

36. Wakabayashi I. Influence of gender on the association of alcohol drinking with blood pressure. Am J Hypertens 2008; 21(12):1310-1317.

37. Barroso SG, Abreu VG, Francischetti EA. A participação do tecido adiposo visceral na gênese da hipertensão e doença cardiovascular aterogênica. Um conceito emergente. Arq. Bras. Cardiol 2002; 78(6);618-630.

38. Movahed MR, Sattur S, Hashemzadeh M. Independent association between type 2 diabetes mellitus and hypertension over a period of 10 years in a large inpatient population. Clin Exp Hypertens 2010; 32(3):198201.

39. Barbosa JB, Silva AAM, Santos AM, Monteiro Júnior FC, Barbosa MM, Barbosa MM, Figueiredo Neto JA, Soares NJS, Nina VJS, Barbosa JN. Prevalência da hipertensão arterial em adultos e fatores associados em São Luís - MA. Arq Bras Cardiol 2008; 91(4):260-266.

40. Sanjuliani AF. Fisiopatologia da hipertensão arterial: conceitos teóricos úteis para a prática clínica. Rev SOCERJ 2002; 15(4):210-218.

41. Lindhorst J, Alexander N, Blignaut J, Rayner B. Differences in hypertension between blacks and whites: an overview. Cardiovasc J Afr 2007; 18(4):241-247.

42. Salomaa V. Genetics of blood pressure. Duodecim 2014; 130(11):1099-1107.

43. Natekar A, Olds RL, Lau MW, Min K, Imoto K, Slavin TP. Elevated blood pressure: our family's fault? The genetics of essential hypertension. World J Cardiol 2014; 6(5):327-337.

44. Kimura L. Fatores genéticos associados à hipertensão essencial em populações remanescentes de quilombos do Vale do Ribeira - São Paulo [tese]. São Paulo: Universidade de São Paulo; 2010.

45. Chor D, Lima CRA. Aspectos epidemiológicos das desigualdades sociais em saúde no Brasil. Cad Saude Publica 2005; 21(5):1586-1594. 
